\title{
Meat consumption in Scotland: analysis from the Expenditure and Food Survey
}

\author{
K. L. Barton ${ }^{1}$, W. L. Wrieden ${ }^{2}$, J. Armstrong ${ }^{3}$ and A. Sherriff ${ }^{4}$ \\ ${ }^{1}$ Centre for Public Health Nutrition Research, University of Dundee, Dundee DDI 9SY, UK, ${ }^{2}$ Public Health Nutrition \\ Research Group, University of Aberdeen, Aberdeen AB25 2ZD, UK, ${ }^{3}$ School of Life Sciences, Glasgow Caledonian \\ University, Glasgow G4 OBA, UK and ${ }^{4}$ Dental Public Health, University of Glasgow, Glasgow G2 3JZ, UK
}

The World Cancer Research Fund (WCRF) report of $2007^{(1)}$ recommended that the population average consumption of cooked red meat should not be $>300 \mathrm{~g}(11 \mathrm{oz}) /$ week, equivalent to a mean of approximately $43 \mathrm{~g} / \mathrm{d}$, of which very little if any should be processed. They define processed meat as that preserved by smoking, curing or salting or addition of chemical preservatives, including that contained in processed foods. Using this definition household food purchase data from the UK Expenditure and Food Survey was re-analysed to estimate red and processed meat consumption in Scotland over the period 2001-2 to 2006 and by Scottish index of multiple deprivation (SIMD) for the period 2001-2 to 2003-4. Adjustments were made for waste (new values derived from the Department for Environment, Food and Rural Affairs ${ }^{(2)}$ and the Waste and Resource Action Programme survey of $2007^{(3)}$ ), meat content of prepared dishes and cooking losses. Data were analysed using general linear models within the complex samples module of SPSS (SPSS Inc., Chicago, IL, USA) weighting to the Scottish population and taking account of sampling methods. Results are presented as population means (i.e. includes consumers and non-consumers), as eaten, for household and eating out foods combined.

\begin{tabular}{|c|c|c|c|c|c|c|c|c|c|}
\hline & & $\begin{array}{c}2001-2 \\
(n 619) \\
(5015 \text { weighted })\end{array}$ & $\begin{array}{c}2002-3 \\
(n \text { 585) } \\
(4967 \text { weighted) }\end{array}$ & $\begin{array}{c}2003-4 \\
(n \text { 546) } \\
\text { (4952 weighted) }\end{array}$ & $\begin{array}{c}2004-5 \\
(n \text { 590) } \\
(4948 \text { weighted })\end{array}$ & $\begin{array}{c}2005-6 \\
(n 566) \\
\text { (4939 weighted) }\end{array}$ & $\begin{array}{c}2006 \\
(n 577) \\
\text { (4906 weighted) }\end{array}$ & $\begin{array}{c}P \text { for } \\
\text { overall } \\
\text { association }\end{array}$ & $\begin{array}{c}P \text { for } \\
\text { linear } \\
\text { association } \\
\end{array}$ \\
\hline $\begin{array}{l}\text { Red meat* } \\
\quad(\mathrm{g} \text { per person per } \mathrm{d})\end{array}$ & Mean & $\begin{array}{l}36.2 \\
335.38 .8\end{array}$ & 36.6 & 36.1 & $\begin{array}{l}34.4 \\
318360\end{array}$ & $\begin{array}{l}34.6 \\
32.370\end{array}$ & 34.4 & 0.737 & 0.143 \\
\hline $\begin{array}{l}\text { Processed red meat } \\
\quad(\mathrm{g} \text { per person per } \mathrm{d})\end{array}$ & $\begin{array}{l}\text { Mean } \\
95 \% \mathrm{CI}\end{array}$ & $\begin{array}{c}26.8 \\
24.2,29.4\end{array}$ & $\begin{array}{c}26.4 \\
24.6,28.1\end{array}$ & $\begin{array}{c}28.5 \\
26.6,30.5\end{array}$ & $\begin{array}{c}25.3 \\
23.5,27.1\end{array}$ & $\begin{array}{c}26.1 \\
24.1,28.1\end{array}$ & $\begin{array}{c}24.3 \\
22.5,26.2\end{array}$ & 0.141 & 0.097 \\
\hline $\begin{array}{l}\text { Total red meat }(\mathrm{g} \text { per } \\
\text { person per } \mathrm{d})\end{array}$ & $\begin{array}{c}\text { Mean } \\
95 \% \text { CI }\end{array}$ & $\begin{array}{c}63.0 \\
58.4,67.6\end{array}$ & $\begin{array}{c}63.0 \\
59.3,66.7\end{array}$ & $\begin{array}{c}64.7 \\
60.7,68.6\end{array}$ & $\begin{array}{c}59.7 \\
55.9,63.4\end{array}$ & $\begin{array}{c}60.7 \\
57.0,64.3\end{array}$ & $\begin{array}{c}58.7 \\
55.0,62.4\end{array}$ & 0.301 & 0.068 \\
\hline
\end{tabular}

*Unprocessed meat included meat products such as beef burgers and sausage rolls, which were mainly unpreserved.

\begin{tabular}{|c|c|c|c|c|c|c|c|c|}
\hline & & \multicolumn{5}{|c|}{ SIMD quintile* } & \multirow{2}{*}{$\begin{array}{l}P \text { for overall } \\
\text { association }\end{array}$} & \multirow{2}{*}{$\begin{array}{l}P \text { for linear } \\
\text { association }\end{array}$} \\
\hline & & 1 & 2 & 3 & 4 & 5 & & \\
\hline $\begin{array}{l}\text { Red meat (g per person } \\
\text { per d) }\end{array}$ & $\begin{array}{l}\text { Mean } \\
95 \% \text { CI }\end{array}$ & $\begin{array}{c}37.6 \\
34.3,40.9\end{array}$ & $\begin{array}{c}35.2 \\
32.8,37.6\end{array}$ & $\begin{array}{c}40.5 \\
36.6,44.5\end{array}$ & $\begin{array}{c}34.8 \\
31.6,38.1\end{array}$ & $\begin{array}{c}33.4 \\
30.1,36.8\end{array}$ & 0.081 & 0.093 \\
\hline $\begin{array}{l}\text { Processed red meat ( } \mathrm{g} \\
\text { per person per } \mathrm{d} \text { ) }\end{array}$ & $\begin{array}{l}\text { Mean } \\
95 \% \text { CI }\end{array}$ & $\begin{array}{c}31.4 \\
29.1,33.7\end{array}$ & $\begin{array}{c}28.7 \\
26.0,31.3\end{array}$ & $\begin{array}{c}26.8 \\
24.4,29.1\end{array}$ & $\begin{array}{c}23.9 \\
21.5,26.3\end{array}$ & $\begin{array}{c}25.4 \\
22.6,28.1\end{array}$ & $<0.001$ & $<0.001$ \\
\hline $\begin{array}{l}\text { Total red meat ( } \mathrm{g} \text { per } \\
\text { person per } \mathrm{d})\end{array}$ & $\begin{array}{l}\text { Mean } \\
95 \% \mathrm{CI}\end{array}$ & $\begin{array}{c}69.0 \\
64.3,73.7\end{array}$ & $\begin{array}{c}69.0 \\
59.5,68.2\end{array}$ & $\begin{array}{c}67.3 \\
61.8,72.7\end{array}$ & $\begin{array}{c}58.7 \\
54.0,63.5\end{array}$ & $\begin{array}{c}58.8 \\
54.1,63.5\end{array}$ & 0.014 & 0.001 \\
\hline
\end{tabular}

*Combined data for years 2001-2 to 2003-4; 1, most deprived; 5, least deprived.

The population estimate for total red meat consumption was $46 \%$ higher than the WCRF recommended population average and there was no significant difference over the 5 years. There was a significant decreasing linear trend with increasing affluence for processed and total red meat $(P<0.001$ and $P=0.001$ respectively for linear trend).

In conclusion, current intakes are higher than desirable for cancer prevention based on the WCRF recommendations. This issue needs to be addressed in future prevention work.

Funding provided by Food Standards Agency Scotland is gratefully acknowledged (Project no. S14035). Data provided by the Department for the Environment, Food and Rural Affairs, Scottish Neighborhood Statistics, the Office of National Statistics and the UK Data Archive.

1. World Cancer Research Fund/American Institute for Cancer Research (2007) Food, Nutrition, Physical Activity, and the Prevention of Cancer: a Global Perspective. Washington, DC: AICR.

2. Department of Environment, Food and Rural Affairs/Office for National Statistics (2008) Family Food. A Report on the 2007 Expenditure and Food Survey. London: The Stationery Office.

3. Waste and Resource Action Programme (2007) The food we waste. http://www.wrap.org.uk/retail/case_studies_research/report_the_food_we.html 